\title{
Zum Sprachverständnis in der Migrationsdebatte in Deutschland ${ }^{1}$
}

\author{
Christoph Schroeder ${ }^{2}$
}

Hinter der öffentlichen Debatte um die sprachliche Vielfalt in Deutschland verbirgt sich oft ein Sprachverständnis, in dem der Begriff Sprache anstelle von Inhalten oder ethnischen oder sozialen Zuordnungen verwendet wird, oder der Wunsch nach der Messbarkeit der persönlichen Integrationsleistung des Zuwanderers oder der Zuwanderin die Sprache zum „Schlüssel“ instrumentalisiert. Diesem problematischen Sprachverständnis setze ich eine Beschreibung der gesellschaftlichen Mehrsprachigkeit in Deutschland und der daraus bereits gezogenen bildungspolitischen Konsequenzen entgegen, gemeint als Beitrag zur Entspannung der Diskussion um die sprachliche Vielfalt in Deutschland.

\section{Verhandlung von Sprache im Kontext von Migration und Integration: Vier Beispiele}

Erstes Beispiel: In Berlin-Neukölln hat vor einigen Jahren ein Laden für islamische Bekleidung aufgemacht. Das ist an sich nichts Besonderes; diese Läden gibt es allerorten, meist erkennt man sie gar nicht, weil sie recht unscheinbar sind und nur auf Türkisch oder in arabischer Schrift eine kleine Inschrift darauf hinweist, was verkauft wird. Bei diesem Laden war es etwas anderes: „Islamische Boutique“ stand in großen Lettern über dem Eingang - und zwischen den Schaufenstern war die Koransure 24:31 zitiert, die den Frauen gebietet, sich gegenüber dem Mann zu verhüllen: in deutscher Sprache, auf großen Lettern, Gold auf Schwarz.

Heinz Buschkowsky, bekannter (inzwischen ehemaliger) Bezirksbürgermeister von Neukölln, fand das problematisch. Mit der Koransure werde, ich zitiere aus der Berliner Zeitung vom 5. September 2011, eine ,sehr orthodoxe Sichtweise des Korans plakativ in den öffentlichen Raum getragen“, und das sei der Integration „nicht dienlich“. Dass das Koranzitat in deutscher Sprache widergegeben wird, das setzt für ihn „noch einen drauf“.

Nicht dass Deutsch verwendet wird, ist das Problem, sondern was auf Deutsch verhandelt wird. Daraus spricht die enttäuschte Hoffnung, dass mit dem Sprachwechsel zum Deutschen bei den Zugewanderten möglichst auch eine Akkulturalisierung einhergehe.

Zweites Beispiel: Wie bekannt haben die Bundesländer in ihrer Kulturhoheit unterschiedliche Verteilerschlüssel beschlossen, dem entsprechend Schulen mit einem spezifisch benachteiligten Schülerklientel finanziell besonders gefördert werden. Eine Reihe von Bundesländern verwendet das Attribut „mit Migrationshintergrund“ als Indikator für eine besondere Belastung der Schule $;^{3}$ andere Bundesländer fragen nur oder auch zusätzlich nach dem wirtschaftlichen Status der Eltern, für dessen Bestimmung die Anträge auf

\footnotetext{
${ }^{1}$ Publicado em: Schroeder, Christoph. Zum Sprachverständnis in der Migrationsdebattein Deutschland. In: Ekinci, Yüksel; Montanari, Elke; Selmani, Lirim (Hrsg.). Grammatik und Variation. Festschrift für Ludger Hoffmann zum 65. Geburtstag. Heidelberg: Synchron, 2017. p. 361-367.

${ }^{2}$ Doutor pela Universidade de Bremen e Habilitação na área de Linguística pela Universidade de Osnabrück . Professor da área de Alemão como Segunda Língua e Língua Estrangeira, do Instituto de Germanística da Universidade de Potsdam, Alemanha.

${ }^{3}$ Dabei definieren die statistischen Landesämter und die Schulämter „Migrationshintergrund“ teilweise unterschiedlich manchmal müssen beide Elternteile zugewandert sein, manchmal nur ein Elternteil, damit das Kriterium „Migrationshintergrund“ für das Kind als zutreffend angesehen wird.
} 
Lehrmittelfreiheit (Lmb) herangezogen werden. Das Bundesland Berlin hat sich dafür entschieden, die Lehrmittelbefreiung und den Indikator ,nichtdeutsche Herkunftssprache (ndH) einzusetzen: Bei der Einschulung werden die Eltern nach der vom Kind in der Familie vorwiegend verwendeten Sprache gefragt, und wenn dies eine andere Sprache als Deutsch ist, dann geht das Kind als „,ndH-Schüler/in“, also als „Schüler oder Schülerin mit nichtdeutscher Herkunftssprache“ in die Statistik der Schule ein. Schulen mit mehr als 40\% ndH-Schülern bekommen mehr Lehrerstunden; ebenso wie Schulen mit mehr als 40\% Lmb-Schülern.

Interessant ist nun, dass die ndH-Quoten aller Schulen auf den Internetseiten der Senatsverwaltung für Bildung veröffentlicht werden - und diese Seiten sind die meistbesuchten Seiten der Senatsverwaltung (Vasilyeva 2013). Die Lmb-Quoten dagegen bleiben unter Verschluss. So konstruiert der Berliner Senat die „nichtdeutsche“ Familiensprache - und damit Mehrsprachigkeit - zu einem zentralen Problemfall der Schule. ${ }^{4}$

Drittes Beispiel: Bleiben wir bei der „Herkunftssprache“. Dieser Begriff hat inzwischen weitestgehend den Begriff der „Muttersprache“ abgelöst, wenn von den ,,anderen“ Sprachen neben dem Deutschen die Rede ist, mit denen mehrsprachige Kinder in Deutschland aufwachsen. Offenbar hat sich die Erkenntnis durchgesetzt, dass der Begriff „Muttersprache“ ein viel zu ungenaues Konstrukt ist, das auf vielerlei verweist, vom "Erwerb“ („Erstsprache“) über die Ethnie, die Nation und die Kompetenz (,muttersprachliches Niveau“) bis hin zum Blut („Mutter“), vgl. Skutnabb-Kangas \& Phillipson (1989). „Herkunftssprache“ dagegen scheint auf den ersten Blick eindeutiger. Der Begriff verweist auf „Herkunft“ - aber worauf verweist das wiederum? Was ist mit dem „Herkommen“ gemeint, mit dem sich „Herkunft“ so offensichtlich verbindet? Wenn damit eine bestimmte soziale Domäne gemeint ist, nämlich das „Zuhause“, dann hätte man sich auch für „Familiensprache“ entscheiden können - hat man aber nicht. „Herkunftssprache“ ist vielmehr „,eine Sprache, die dort gesprochen wird, wo jemand herkommt" - und das ist entweder geographisch oder ethnisch oder national gemeint, verweist aber keinesfalls auf den Ort, wo der Sprecher oder die Sprecherin dieser Sprache, auf die damit referiert wird, lebt und Teil der Gesellschaft ist, nämlich Deutschland. ${ }^{5}$

Viertes Beispiel: Das eingängige Schlagwort von der „Sprache als Schlüssel zur Integration" begleitet die beeindruckende Maschinerie der Integrationskurse, die im Zuge der Umsetzung des Zuwanderungsgesetzes von 2005 eingerichtet wurden. Tatsächlich bietet Sprache sich, wie Bommes (2006) aufzeigt, aus der Sicht des Staates hervorragend als ordnungspolitisch nutzbarer Indikator für Integrationswilligkeit an:

- Zum Einen kann der Staat bezüglich Sprache ein Angebot machen und gleichzeitig die Verantwortung für die Nichtannahme des Angebots den Zugewanderten selbst zuschreiben: Wer das Angebot des Integrationskurses nicht annimmt, kann individuell für sein Verhalten haftbar gemacht werden, indem aufenthaltsrechtliche Konsequenzen gezogen oder auch staatliche Hilfeleistungen gekürzt werden. Wirtschaftliche Selbstständigkeit, ein anderer starker Integrationsindikator, ist dagegen - aus der Sicht des Staates - wesentlich problematischer in der Anwendung als Kriterium, denn Arbeit kann man den

\footnotetext{
${ }^{4}$ Nicht zuletzt die Rezeption der ndH-Quoten hat in Berlin dramatische Segregationstendenzen in den Grund- und Sekundarschulen ausgelöst, die unlängst in einer Studie des Sachverständigenrats deutscher Stiftungen für Integration und Migration (SVR 2012) nachgewiesen wurden. Siehe im Übrigen die funktional-semantische Analyse von Migrationshintergrund bei Scarvaglieri \& Zech (2013), die ganz ähnlich wie „ndH“ eben soziale Exklusionen perpetuiert nur eben nicht explizit auf der Grundlage von Sprache wie bei „ndH“.

${ }^{5}$ Der Verweis nach „draußen“ bei „Herkunftssprache“ findet umgekehrt auch darin seinen Ausdruck, dass im Kontext der in Deutschland (landes-)verfassungsgemäß anerkannten regionalen Minderheitensprachen Dänisch, Sorbisch und Friesisch eben nicht von „Herkunftssprachen“ gesprochen wird.
} 
Neuzuwanderern nicht ohne Weiteres anbieten. Eben so wenig kann der Staat soziale Kontakte anbieten oder das Zustandekommen sozialer Kontakte regulieren.

- Zum Zweiten ist Sprache bzw. Sprachkompetenz messbar - jedenfalls dann, wenn man unter Sprache ein endliches/abgeschlossenes und standardisierbares Regelund Formenwerk versteht und mit ihr keinen Handlungsbegriff verbindet. Mittels eines Sprachtests ist valide, nachprüfbar und wiederholbar feststellbar, ob jemand die deutsche Sprache auf einem bestimmten Level beherrscht oder nicht. Ähnliches wäre in Bezug auf soziale und wirtschaftliche Integration nicht denkbar oder zumindest hochproblematisch.

Natürlich sind beide Argumente aus wissenschaftlicher Sicht sehr anzweifelbar, aber das spielt dann keine Rolle, wenn wir uns die mögliche Sicht des Staates zu eigen machen, der die Zuwanderung und Integration in einer Form regulieren will, die ihn als erfolgreichen Agenten zeigt.

\section{Mehrsprachigkeit und ihre sozialen Räume in Deutschland}

In den vier Beispielen verbindet sich mit dem Begriff der Sprache jeweils etwas anderes als Sprache selbst: Im ersten Beispiel steht die inhaltliche Zuschreibung im Vordergrund; im zweiten Beispiel führt die individuelle Mehrsprachigkeit zur Konstruktion einer Leistungsschwäche und eines niedrigen Bildungshintergrunds. Im dritten Beispiel verweist das erste Glied des Kompositums auf eine geographische, ethnische oder nationale Herkunft außerhalb dieser Gesellschaft und im vierten Beispiel sind es die Handhabbarkeit, die individuelle Überantwortbarkeit und die Messbarkeit, die Sprache als zentralen Indikator für Integrationswilligkeit tauglich erscheinen lassen.

Diese Zuschreibungen und Instrumentalisierungen trüben den Blick auf die Mehrsprachigkeitsverhältnisse; sie spiegeln den Versuch eines öffentlichen Diskurses, die Mehrsprachigkeitsverhältnisse zu verschleiern, um sie nicht als Faktoren in sprachenpolitischen Entscheidungen zulassen zu müssen.

Für einen entspannteren Blick auf Mehrsprachigkeit und die sprachenpolitischen Maßnahmen, die sie regulieren soll(t)en, müssen wir zunächst einmal die mehrsprachigen Verhältnisse, die wir in Deutschland haben, kurz unter die Lupe nehmen:

Deutschland ist ein de facto mehrsprachiges Land, weil die Menschen die hier wohnen, viele unterschiedliche Sprachen sprechen - das zeigen die Surveys, die in den vergangenen Jahren in Essen (Chlosta et al. 2003), Hamburg (Fürstenau et al. 2003), Freiburg (Decker \& Schnitzer 2012) und zuletzt auch in Thüringen (Ahrenholz \& Maak 2013) durchgeführt wurden. Die gesellschaftliche Mehrsprachigkeit in Deutschland ist dadurch charakterisiert, dass es viele nicht regional gebundene Minderheitensprachen gibt, von denen einige Sprecherzahlen haben, die bis in die Millionen gehen, z.B. Russisch, Polnisch und Türkisch. Diese Sprachen legen eine erstaunliche (weil politisch-gesellschaftlich unerwartete) Vitalität an den Tag, die sich stabilisiert: Es haben sich Sprachgemeinschaften etabliert. Sie haben sich mit der Zuwanderung gebildet, und die Sprachen haben keinen expliziten verfassungsrechtlichen Status, anders als die zahlenmäßig wesentlich kleineren, regional gebundenen Minderheitensprachen (Friesisch, Dänisch und Sorbisch) und die nach der europäischen Charta der Minderheitensprachen anerkannten Sprachen Romanes und die Regionalsprache Niederdeutsch.

Die durch Zuwanderung entstandene gesellschaftliche Mehrsprachigkeit in Deutschland ist nach Maas (2008: 41ff.) weiterhin dadurch charakterisiert, dass die Verwendung der Sprachen auf bestimmte soziale Räume beschränkt ist, und das wiederum hat Auswirkungen auf die verwendeten Varietäten: 
- Die vielen ,,anderen“ Sprachen werden neben Deutsch vor allem im informellen privaten sozialen Raum gesprochen, in der Familie, mit Freunden - und sie sind vorrangig mündlich.

- Ein weiterer Raum, in dem andere Sprachen neben Deutsch verwendet werden, ist der informell-öffentliche Raum, sozusagen der „Markt“/der „Kiez“. Allerdings ist hier auch schon die Verwendung anderer Sprachen als Deutsch mit Unbekannten gewissermaßen „Aushandlungssache“; die Default-Sprache in diesem sozialen Raum ist zumeist das Deutsche. Die Varietät des Deutschen, die hier verwendet wird, ist die regional geprägte Umgangssprache, auch die anderen Sprachen werden in ihren umgangssprachlichen Varietäten gesprochen - und es gibt faszinierende, auch identitätsstiftende, Hybriditäten zwischen den Sprachen, die eine strukturelle Festigkeit als Ethnolekte und Jugendsprachen erlangen (Selting 2011, Wiese 2012). In diesen Raum gehören aber auch die Medien und das Internet, oder allgemein der Gebrauch von Schriftsprache in der realen oder der virtuellen Öffentlichkeit. Hier dominiert das Deutsche, allerdings etabliert sich auch ein vielfältiger Medien- und Kulturbereich in anderen Sprachen, auch ein gemischtsprachiger (Androutsolopous 2013).

- Der zentrale soziale Raum, der ausschließlich dem Deutschen vorbehalten ist, ist der der gesellschaftlichen Einrichtungen und Institutionen, also der Einrichtungen, mit denen die Gesellschaft sich selbst „erschafft“ und die Ausdruck ihrer Arbeitsteilung sind: Schulen, Ämter, Versorgungseinrichtungen, der Geschäftsverkehr uvm.: Wer hier lebt und selbständig, also ohne Hilfe von Übersetzern, an der Gesellschaft partizipieren will, wer sie also mitschaffen will, wird Deutsch lernen müssen, und zwar die schriftsprachlich orientierte Varietät des Deutschen.

- Ein vierter sozialer Raum, in dem wiederum durchaus andere Sprachen als Deutsch eine Gültigkeit haben, und zwar diesmal auch schriftsprachlich orientiert, ist der der international operierenden und der transnationalen Einrichtungen.

\section{Sprachen als Schlüssel zur Bildung}

Im Sinne des oben Ausgeführten hat „Integration durch Sprache“ vorrangig zu bedeuten, dass alle in der Gesellschaft lebenden Menschen dazu befähigt werden, diejenigen sprachlichen Mittel in die Hand zu bekommen, mittels derer sie an der Gesellschaft partizipieren können - und das ist in Deutschland eine spezifische Form oder Varietät des Deutschen, das schriftsprachlich orientierte Deutsch. Alles andere findet sich von selbst, gehört zumindest nicht in die Verantwortlichkeit der staatlichen Institutionen. So ist das Wort von der „Sprache als Schlüssel zur Integration“ (siehe viertes Beispiel) zu gewaltig. Gemeint sein und getestet werden kann nur diese spezifische Varietät des Deutschen, alles andere ist Privatsache!

Gleichzeitig müssen wir uns darüber im Klaren sein, dass die gelebte Mehrsprachigkeit bleiben wird, weil sie sich biographisch einschreibt und nicht etwas ist, dessen man sich beliebig entledigen kann. In dieser Vielfalt der sprachlichen Verhältnisse haben sich die Sprachen und Sprachlichkeiten längst den inhaltlichen (siehe erstes Beispiel) und den ethnischen, geographischen oder nationalen (siehe drittes Beispiel) Zuschreibungen entzogen. Auch wissen wir, dass Mehrsprachigkeit und niedriger Bildungserfolg zwar unter bestimmten institutionell verantworteten Bedingungen ko-präsent sind, ähnlich wie Lehrmittelbefreiung und niedriger Bildungserfolg, aber wir wissen auch, dass es völlig absurd wäre, von einem auf das andere zu schließen (siehe zweites Beispiel).

Weil inzwischen mehr als ein Drittel der Kinder und jungen Leute in Deutschland mehrsprachig sind und bleiben, ist es selbstverständlich, dass die Bildungsinstitutionen in der 
Primar- und der Sekundarbildung darauf reagieren (müssen), indem sie es als Ressource nutzen und fördern, im Sinne einer mancherorts bereits praktizierten ganzheitlichen Sprachbildung. Und was hier sinnvoll ist, wissen wir auch:

- Im Kindergarten führen ein früher Kindergartenbesuch in Kombination mit einem Angebot zu präliterater und family literacy- Unterstützung auch der Familiensprache zu guten Ergebnissen (Lesemann et al. 2001).

- In der Grundschule, die eine Ganztagsschule ist, beginnt sich glücklicherweise das Verständnis vom Deutschunterricht in allen Fächern durchzusetzen. Dies ist mit bilingualer Alphabetisierung vor allem bei Dominanz der Familiensprache zu verbinden (Reich 2011).

- In den weiterführenden Schulen benötigen wir darüber hinaus ein verstärktes Angebot von in Deutschland gesprochenen Sprachen als zweite Fremdsprachen nach Englisch, und zwar als Angebot für alle Schülerinnen und Schüler, damit der sogenannte „Herkunftssprachenunterricht" endlich aus seinem Nischendasein befreit wird. Es kann den Lehrerinnen und Lehrern durchaus zugemutet werden, binnendifferenziert zu arbeiten, wenn fortgeschrittene Sprecherinnen und Sprecher der jeweiligen Sprachen mit im Unterricht sitzen (Schroeder \& Küppers 2016).

- Für den Anfangsunterricht im Deutschen, der derzeit aufgrund der verstärkten Zuwanderung durch Flucht in aller Munde ist, ist die Bildung von Deutschfördergruppen sinnvoll, wobei klare curriculare Lernzielvorgaben auf die frühe Integration in den Regelunterricht abzielen.

- Bei den Integrationskursen, deren Einrichtung natürlich ein großer Schritt vorwärts ist in Richtung auf eine „Willkommenskultur“, bedarf es einem Abrücken von der Einheitlichkeit und der Prüfungsorientierung der Kurse, um den hohen Druck auf die vielen lernungewohnten Teilnehmer und Teilnehmerinnen, der für die vielen Abbrüche mitverantwortlich ist, etwas abzumildern und auf die Heterogenität der Teilnehmererwartungen zu reagieren (Schroeder \& Zakharova 2015). Sinnvoll wäre es, eine Diversifizierung des Kursangebots mit der Vergabe eines „DeutschkursGutscheins“ zu verbinden, der es jedem Zuwanderer ermöglicht, aus einem breiten Kursportfolio ein seinen Bedürfnissen entsprechendes Kursangebot im Umfang von 600 Stunden zu wählen; über diese Stundenzahl hinausgehende Kurse können auf Kreditbasis (ähnlich BAföG) belegt werden.

Last but not least benötigen wir eine hervorragende Ausbildung all derer, die in den verschiedenen Bildungseinrichtungen und Kursen tätig sind: Ein entspannter, situationsangemessener Blick auf Mehrsprachigkeit und die sprachliche Vielfalt in Deutschland basiert vor allem auf Wissen und Erfahrung.

\section{Literatur}

Ahrenholz, Bernt \& Diana Maak. 2013. Zur Situation von SchülerInnen nicht-deutscher Herkunftssprache in Thüringen unter besonderer Berücksichtigung von SeiteneinsteigernAbschlussbericht zum Projekt „, Mehrsprachigkeit an Thüringer Schulen (MaTS) “., durchgeführt im Auftrage des TMBWK (Online unter: http://www.dazportal.de/images/Berichte/bm_band_01_mats_bericht_20130618_final.pdf, Zugriff: 30.10.2015)

Androutsopoulos, Jannis. 2013. Code-switching in computer-mediated communication. In: Susan C. Herring, Dieter Stein \& Tuja Virtanen (eds). Pragmatics of computer-mediated communication. Berlin: de Gruyter, 667-694. 
Bommes, Michael. 2006. Integration durch Sprache als politisches Konzept. In: Ulrike Davy \& Albrecht Weber (Hrsg.). Paradigmenwechsel in Einwanderungsfragen? Überlegungen zum neuen Zuwanderungsgesetz. Baden-Baden: Nomos, 59-86.

Chlosta, Christoph, Torsten Ostermann \& Christoph Schroeder. 2003. Die

„Durchschnittsschule” und ihre Sprachen, Ergebnisse des Projekts Sprachenerhebung Essener Grundschulen (SPREEG). Essener Linguistische Skripte (ELiS_e) 1, 43-139. (Online http://www.elise.uni-essen.de/elise01_2003.html, Zugriff: 30.10.2015).

Decker, Yvonne, \& Katja Schnitzer. 2012. FreiSprachen - Eine flächendeckende Erhebung der Sprachenvielfalt an Freiburger Grundschulen. In: Bernt Ahrenholz \& Werner Knapp (Hrsg.). Sprachstand erheben - Spracherwerb erforschen. Beiträge aus dem 6. Workshop "Kinder mit Migrationshintergrund", 2010. Stuttgart: Fillibach bei Klett, 95-112. Fürstenau, Sara, Ingrid Gogolin \& Kutlay Yağmur (Hrsg.). 2003. Mehrsprachigkeit in Hamburg. Ergebnisse einer Sprachenerhebung an den Grundschulen in Hamburg. Münster: Waxmann.

Lesemann, Paul M. \& Cathy van Tuijl. 2001. Home support for bilingual development of Turkish 4-6-year-old immigrant children in the Netherlands. Efficacy of a home-based educational programme. Journal of Multilingual and Multicultural Development (JMMD) 4, 309-324.

Maas, Utz. 2008. Sprache und Sprachen in der Migrationsgesellschaft. Die schriftkulturelle Dimension. (IMIS-Schriften 15). Göttigen: V \& R Unipress.

Selting, Margret. 2011. Prosody and unit-construction in an ethnic style: the case of Turkish German and its use and function in conversation. In: Friederike Kern \& Margret Selting (eds.). Ethnic styles of speaking in European Metropolitan Areas. Amsterdam: Benjamins, 131-159.

Reich, Hans R. 2011. Schriftsprachliche Fähigkeiten türkisch-deutscher Grundschülerinnen und Grundschüler in Köln. Stand: 12/2011. Ein Untersuchungsbericht, herausgegeben von der Bezirksregierung Köln. (Online http://www.bezregkoeln.nrw.de/brk_internet/organisation/abteilung04/dezernat_41/broschuere_sprachstark.pdf, Zugriff: 12.09.2013)

Sachverständigenrat deutscher Stiftungen für Integration und Migration (Hrsg.). 2012. Segregation an Grundschulen. Der Einfluss der elterlichen Schulwahl. Policy Brief. Berlin.

Scarvagliere, Claudo \& Claudia Zech. 2013. ,ganz normale Jugendliche, allerdings meist mit Migrationshintergrund“. Eine funktional-semantische Analyse von „Migrationshintergrund. Zeitschrift für Angewandte Linguistik, 201-227.

Schroeder, Christoph \& Almut Küppers. 2016. Türkischunterricht im deutschen Schulsystem: Bestandsaufnahme und Perspektiven. In: Barbara Pusch, Almut Küppers \& Pinar UyanSemerci (eds.). Education in transnational spaces. Theory, practice and research based on German-Turkish examples. Berlin: Springer VS, 191-212.

Skutnabb-Kangas, Tove \& Robert Phillipson. 1989. 'Mother Tongue': The theoretical and sociopolitical construction of a concept. In: Ulrich Ammon (ed.). Status and function of languages and language varieties. Berlin: de Gruyter, 450-477.

Schroeder, Christoph \& Natalia Zakharova. 2015. Sind die Integrationskurse ein Erfolgsmodell? Kritische Bilanz und Ausblick. Zeitschrift für Ausländerrecht und Ausländerpolitik (ZAR) 8, 257-262.

Wiese, Heike. 2012. Kiezdeutsch - ein neuer Dialekt entsteht. München: C.H. Beck. Vasilyeva, Larisa. 2013. Zum Merkmal der ,nichtdeutschen Herkunftssprache“ (ndH) in der schulischen Sprachförderung im Land Berlin. Masterarbeit, Universität Potsdam. Potsdam: PublishUP (Online https://publishup.uni-potsdam.de/opus4ubp/files/6818/vasilyeva_master.pdf, Zugriff: 30.10.15) 